\title{
Advanced Modulation Scheme for Visible Light Communication
}

\author{
Agha Yasir Ali*, Razia Zia, Lubna Farhi, Sadia Ahmed, Umm E Laila \\ and Muhammad Ibrar-ul-Haque \\ Sir Syed University of Engineering and Technology, Karachi City, Sindh, Pakistan; \\ aghayasirali@hotmail.com, raziamaroof@yahoo.com, Ifarhi@ssuet.edu.pk, \\ sadiah_ahmed@live.com, ulaila@ssuet.edu.pk,mihaque@ssuet.edu.pk
}

\begin{abstract}
In this paper, an improved version of existing modulation schemes is investigated by inserting Hadamard codes (HC) at the peak slot of each symbol for the Visible Light Communication (VLC) system. The performance of the proposed scheme is analyzed and compared with existing schemes On-Off Keying (OOK), Pulse Position Modulation (PPM), Differential PPM (DPPM), Pulse Amplitude Modulation (PAM), Differential Amplitude PPM (DAPPM) and Differential Pulse Interval Modulation (DPIM). Moreover, the symbol error rate (SER) for $k^{\text {th }}$ PAM is derived for $d_{s}$ distances between symbols. The performance in Symbol Error Rate (SER) is improved by introducing the Positive and Negative Amplitudes (PNA) levels with respect to DC-bias. The theoretical and simulation results show that the proposed scheme outperforms the existing schemes in terms of bandwidth, and transmission capacity.
\end{abstract}

Keywords: Hadamard Code Matrix, Modulation, Visible Light Communication

\section{Introduction}

Visible light communication (VLC) has been the object of extensive interest in the past few years. Many potential applications for this technology have been suggested. The choice of the modulation scheme is one of the critical factors of realizing an efficient wireless optical communication system at cost of complexity, power, error performance and bandwidth. Most commonly modulation schemes are adopted in VLC systems are: OOK, PPM, DPPM, PAM, DAPPM and DPIM $\stackrel{1-3}{\text {. }}$.

Several modified modulation schemes have been investigated in the literature under various conditions and network structures. The Low-Density Parity Check (LDPC) coded $4 \times 4$ and $4 \times 8$ DAPPM Free-space Optical Communication (FSO) system is constructed $\mathrm{in}^{4}$. The simulation results show approximately $2 \mathrm{~dB}$ transmits power reduction against classical LDPC-DAPPM without losing Bit Error Rate (BER) performance. BER performance improvement is achieved and the reliability of the FSO system is also enhanced in ${ }^{5}$, by designing gamma- gamma channel applicable decoding. Four-dimensional modulation scheme is proposed in ${ }^{6}$, the colors and PPM schemes are combined in order to decrease the crosstalk between LEDs. Different symbol codification rates are studied in ${ }^{7}$, to improve the Symbol Error Rate (SER) while minimizing the effect over the throughput of the whole system. The analysis of PPM and DPIM in $\frac{8}{\text {, show }}$ that the DPIM scheme is a strong candidate when the Transmission Capacity (TC) and power performances are considered. It is analyzed that high data rate is achieved at the cost of high bandwidth and transmission power.

The proposed modulation scheme achieves the trade-off between flickering and SER performance. The bandwidth efficiency, TC of PPM, DPPM and DAPPM are improved as compared to the reference given in $\frac{1,3,8-10}{3}$. According to the best knowledge of the author, none of the improved and efficient modulation schemes is presented by merging $\mathrm{HCs}$ with existing schemes. The high rate and low bit error rate at the cost of a limited increasing of complexity is achieved in ${ }^{11}$ by merging of Color Shift Keying (CSK) and PPM. Interleaving and overlapping

${ }^{*}$ Author for correspondence 
techniques are introduced to increase the bit-rate by using Expurgated Pulse-Position Modulation (EPPM) schemes in ${ }^{12}$. The Grouped modulation is proposed in $\frac{13}{}$, the multiple-level PAM optical signals are utilized and enhance the data rate up to $50 \mathrm{Mb} / \mathrm{s}$ at 1e-6-bit error rate. The trade-off between dimming loss and transmission rate is achieved in ${ }^{14}$ by using MPPM. The dimming loss is modeled by the quantization loss within the dimming range for the discrete transmission power levels however, the transmission rate is evaluated by the channel capacity. The proposed technique can be applied to all existing techniques mentioned in the literature.

In this paper, the properties of PPM, DPPM, DAPPM, DPIM and PAM have been analyzed with Hadamard Code (HC), and every HC has a different bandwidth which can be measured from the shortest time of peak slot as given in $\frac{15}{15}$. The analysis shows that the proposed scheme provides a wider view on the performance for wide range of design parameters. In order to improve SER, the proposed technique does not require an additional power to increase the distance between symbols on a constellation diagram. Therefore, the PNA levels are utilized to maintain average energy $E_{\text {avg }}$.

The half numbers of symbols are above and below from $\mathrm{P}_{\mathrm{dc}}$ on the constellation diagram, and having equal distances on the constellation, as shown in Figure 1. Similarly, the higher values of $k$ can be separated in the same way. However, the larger distances between symbols can cause the flickering problems. Therefore, a tradeoff between SER and flickering is adopted in the room $\frac{16}{}$.

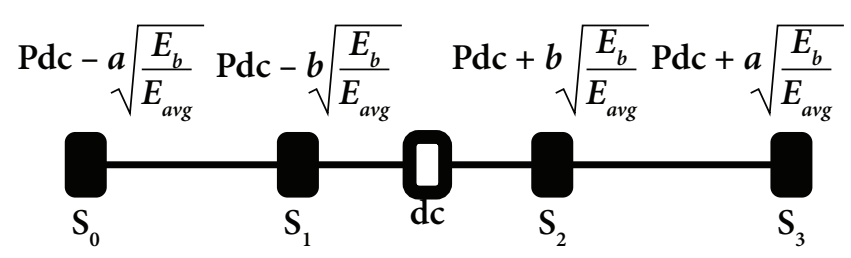

Figure 1. PNA levels for 4 PAM constellations.

This paper is organized as follows. Section 2 explains the proposed system model. The analytical analyses are presented in Section 3. Section 4 express the simulation results. Section 5 concludes this paper.

Table 1. Mapping of 3-bit OOK words with HC into PPM, DPPM, and DAPPM symbols ( $\mathrm{H}_{\text {row }}^{\text {amp }}=$ Hadamard code with amplitude)

\begin{tabular}{|l|l|l|l|l|l|l|}
\hline OOK HC & \multicolumn{2}{|l|}{$\begin{array}{l}\text { PPMHC, } \\
8 \mathrm{~L} / 4 \mathrm{~L}\end{array}$} & \multicolumn{2}{l|}{$\begin{array}{l}\text { DPPMHC, } \\
8 \mathrm{~L} / 4 \mathrm{~L}\end{array}$} & $\begin{array}{l}\text { DAPPMHC, } \\
2 k, 4 \mathrm{~L}\end{array}$ & $\begin{array}{l}\text { DAPPMHC, } \\
4 k, 2 \mathrm{~L}\end{array}$ \\
\hline 000 & $\begin{array}{l}H_{1} 0000 \\
000\end{array}$ & $H_{1} 000$ & $H_{1}$ & $H_{1}$ & $H_{1}$ & $H_{1}$ \\
\hline $00 H_{1}$ & $\begin{array}{l}0 H_{1} 000 \\
000\end{array}$ & $0 H_{1} 00$ & $0 H_{1}$ & $0 H_{1}$ & $0 H_{1}$ & $0 H_{1}$ \\
\hline $0 H_{1} 0$ & $\begin{array}{l}00 H_{1} 00 \\
000\end{array}$ & $00 H_{1} 0$ & $00 H_{1}$ & $00 H_{1}$ & $00 H_{1}$ & $H_{1}^{2}$ \\
\hline $0 H_{1} H_{1}$ & $\begin{array}{l}000 H_{1} 0 \\
000\end{array}$ & $000 H_{1}$ & $000 H_{1}$ & $000 H_{1}$ & $000 H_{1}$ & $0 H_{1}^{2}$ \\
\hline$H_{1} 00$ & $\begin{array}{l}0000 H_{1} \\
000\end{array}$ & $H_{2} 000$ & $0000 H_{1}$ & $H_{2}$ & $H_{1}^{2}$ & $H_{1}^{3}$ \\
\hline$H_{1} 0 H_{1}$ & $\begin{array}{l}00000 H_{1} \\
00\end{array}$ & $0 H_{2} 00$ & $00000 H_{1}$ & $0 H_{2}$ & $0 H_{1}^{2}$ & $0 H_{1}^{3}$ \\
\hline$H_{1} H_{1} 0$ & $\begin{array}{l}000000 \\
H_{1} 0\end{array}$ & $00 H_{2} 0$ & $\begin{array}{l}000000 \\
H_{1}\end{array}$ & $00 H_{2}$ & $00 H_{1}^{2}$ & $H_{1}^{4}$ \\
\hline$H_{1} H_{1} H_{1}$ & $\begin{array}{l}000000 \\
0 H_{1}\end{array}$ & $000 H_{2}$ & $\begin{array}{l}000000 \\
0 H_{1}\end{array}$ & $000 H_{2}$ & $000 H_{1}^{2}$ & $0 H_{1}^{4}$ \\
\hline
\end{tabular}




\section{The Proposed System Model}

LEDs are used to transmit the desired signal by using the most viable modulation is the Intensity Modulation and Direct Detection (IM/DD), in which the desired waveform is modulated into the instantaneous power of the carrier. Modeling a VLC link as a baseband linear, timeinvariant system having impulse response $h(t)$. The VLC channel can be described as a flat fading channel and a diffuse channel, depending on the link conditions. The input pulse $x(t)$ is passing through the Additive White Gaussian Noise (AWGN) $\sigma(t)$ :

$$
y(t)=R h(t) * x(t)+\sigma(t)
$$

$y(t)$ is the photo-detector current, $R$ represents the photo responsivity of the photo-detector (in A/W). The time average transmitted optical power is given by:

$$
P_{t}=\lim _{T \rightarrow \infty} \frac{1}{2 T} \int_{-T}^{T} x(t) d t
$$

The average received optical power generally can be determined as ${ }^{17}$ :

$$
P_{r}=H_{L O S}(0) P_{t(L O S)}+\sum H_{r e f}(0) P_{t(r e f)}
$$

where, $H(0)=\int_{-\infty}^{\infty} h(t) d t$ is the channel DC gain.

$$
H(0)_{L O S}=\left\{\begin{array}{c}
\frac{(l+1) A_{P D} \gamma \cos ^{l}(\varphi) g(\psi) T(\psi)_{c o f} \cos (\psi)}{2 \pi d^{2}} \\
0, \text { elsewhere }
\end{array}\right.
$$

The Line Of Sight (LOS) links and Non-LOS (NLOS) paths are considered. The DC gain on the first reflection is $H(0)_{r e f}$

$$
H(0)_{r e f}=\left\{\begin{array}{c}
\frac{(l+1) A_{P D} * v a}{2 \pi D_{1}^{2} D_{2}^{2}} d A \cos (\alpha) \cos (\beta) \\
0, \text { elsewhere } \\
v a=\gamma \cos ^{l}(\varphi) g(\psi) T(\psi)_{c o f} \cos (\psi) \\
0 \leq \psi \leq \psi_{c}
\end{array}\right.
$$

where, $d$ is the distance between the receiver and transmitter, $D_{1}$ is the distance between transmitter and reflective point $D_{2}$ is the distance between reflective point and receiver, $\gamma$ is the reflectance factor, $d A$ is reflective area of small region, $\alpha$ is the angle of irradiance to the receiver, $\beta$ is the angle of incidence to the receiver, $l$ is the order of Lambertian emission, $A_{P D}$ is the receiving area of photo detector, $\varphi$ is the irradiance angle, $\psi$ is the angle of incidence, $T(\psi)_{\text {cof }}$ is the signal transmission coefficient of an optical filter and $\psi_{c}$ is the Field of View (FOV), $p$ is the refractive index, $g(\psi)$ is the gain of the optical concentrator.

$$
g(\psi)=\left\{\begin{array}{c}
\frac{p^{2}}{\sin ^{2} \psi_{c}}, 0 \leq \psi \leq \psi_{c} \\
0,0 \geq \psi_{c}
\end{array}\right.
$$

The Figure 2 shows that the bit streams are encoded with $\mathrm{HC}_{s}$ and Pulse Modulation Schemes (PMS). Then, symbols pass through transmit filter and DC bias for transmission. After passing the AWGN channel, the signal is received at DC filter. Then, it is followed by the set of matched filters to detect the HCs and passes to Maximum Likelihood (ML) for amplitude $(1,2,3 \ldots k)$ detection. Finally, the signal is decoded.

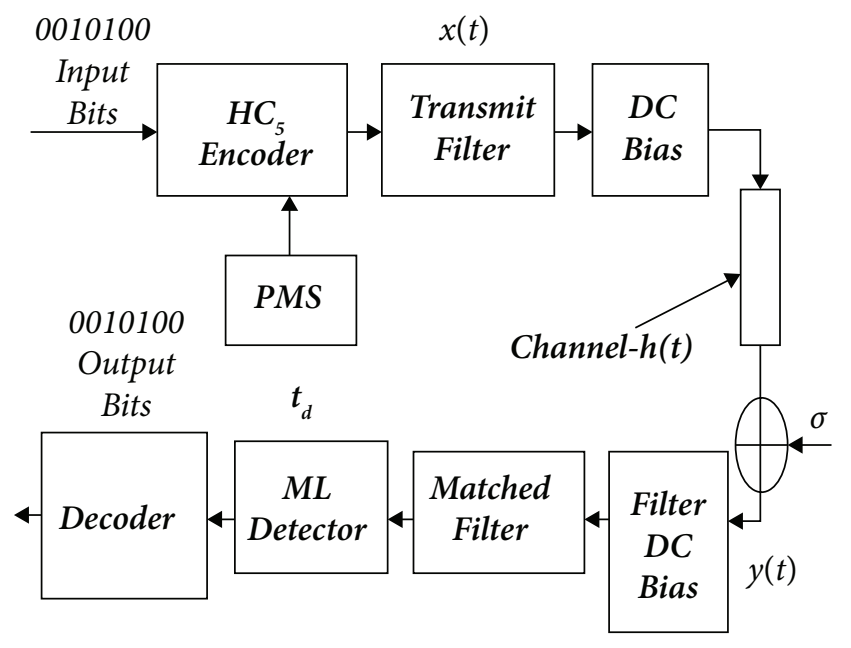

Figure 2. Proposed system model.

The different pulse modulation schemes have their own pros and cons. PPM is power efficient at the cost of bandwidth $\frac{18}{}$. However, PPM with $\mathrm{HC}_{s}$ (PPMHC) is comparatively more efficient with respect to bandwidth and TC and power per bits. Similarly, for the combination of DPPM, DPIM and DAPPM with $H_{c}$ improve the bit rate and TC in time scales at same bandwidth. Table 1 shows the structure of symbol for two HC codes different schemes. In this paper, the perfect synchronization is considered between the transmitter and receiver and there is no Inter-Symbol Interference (ISI). 


\section{Analysis}

\subsection{Pulse Position Modulation with Hadamard Code (PPMHC)}

Consider a case, when the number of possible symbols can be represented as $S_{P P M H C}=L \times H C_{s}$ in PPMHC; where $S_{P P M H C}$ shows the maximum number of symbols, $L$ is the number of slots, and $H C_{s}$ is the number $\mathrm{HC}$. This implies that the bits per symbol $M$ of the scheme can be improved with $L$ and HC, which can be defined as $M=\log _{2}\left(S_{P P M H C}\right)$. In other word, the number of $L$ is reduced by a factor of $1 / H C_{s}$, which can be express as $L_{P P M H C}=2^{M} / H C_{s}$.

However, in multilevel PPMHC (MPPMHC), a symbol can be constructed with the combination of HCs, L and $P_{N o}$, where $P_{\text {No }}$ is number of peak slots in each symbol. If the maximum number of $H C_{s}$ and $P_{N o}$ are used in every symbol then all possible symbols can be expressed as $S_{M P P M H C}=H C_{s} \times\left(\begin{array}{c}L_{P P M H C} \\ P_{N o}\end{array}\right)$ where $H C_{s} \leq(L-1)$ and $P_{N o} \leq(L-1)$, If $P_{N o}$ and $H C_{s}$ are considered from

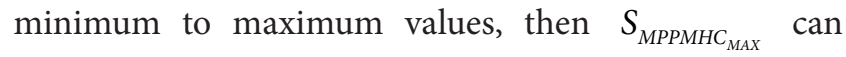
be expressed as: $S_{M P P M H C}=\left(H C_{s}\right)^{2} \times\left(\begin{array}{c}L \\ P_{N o}\end{array}\right)$ where the range of $H C_{s}$ and $P_{N o}$ are $2<H C_{s}<(L-1)$, and $2<P_{N o}<(L-1)$ respectively. However, all possible combination of the symbol cannot be utilized, as 2, 3 and 4 bits per symbol require 4, 8 and 16 number of symbols respectively. Thus, the best possible symbols can be selected based on the hamming distance that provides better SER performance.

\subsection{Differential PPMHC}

The differential PPMHC (DPPMHC) is constructed by removing the empty slots of PPM and putting HCs in peak slots of the symbols. Therefore, the average number of slots per symbol can be defined as $L_{(\text {avg }) D P M H C}=\left(2^{M}+H C_{s}\right) / 2 H C_{s}$. This provides the improving the data throughput and bandwidth efficiency of the system. The number of symbol for DPPMHC is $S_{D P P M H C}=H C_{s} \times L_{(\max ) D P P M H C}$, where $L_{(\max ) D P P M H C}=2^{M} / H C_{s}$. Each DPPMHC symbol ends with the $\mathrm{HC}$ as given in Table 1, thus displaying an inherent symbol synchronization capability at the receiver.
Due to variation in the number of slots of a symbol, the error not only affects the corresponding symbol but also subsequent symbols, thus resulting in multiple symbol errors. The AWGN channel, and any given slots $L$, DPPMHC has the same power requirements per symbol but a slightly higher power requirement in time scale because DPPMHC contains more number of bits at time scale as compared to DPPM, PPM or PPMHC.

Unlike DPPMHC, DPIMHC contains one additional empty slot guard band (GB). Therefore, one or more than one GBs are used depending upon channel condition. The input bit stream is encoded by inserting empty slots in DPPMHC symbol and the bit per symbol is $M=\log _{2}\left(L_{(\max ) D P P M H C} * H C_{s}\right)$.

The DPIMHC has a reduced complexity level as compared to PPMHC and DPPMHC due to its built-in symbol synchronization. The minimum and maximum symbol durations are $T_{s}$ to $T_{s} L_{(\max ) D P P M H C}$, respectively, where $T_{s}$ is the symbol duration. A single $G B(G B=1)$ changes the minimum and maximum symbol durations to $G B+T_{s}$ and $(L+G B) T_{s}$, respectively. Table 1 shows the three bits per symbol by using two pulses $\mathrm{HC}_{2}$ with one GB. The symbol duration and overall data rate are variable. Therefore, the slot duration is chosen such that the mean symbol duration is equal to the time taken to transmit the same number of bits using fixed symbol length schemes such as OOK or PPMHC. This slot duration is given as.

$$
T_{\text {slot }_{\text {PIMHC }}}=\frac{T_{b} M}{L_{D P I M H C}}
$$

where, $L_{\text {DPIMHC }}$ is the average symbol length of DPIMHC, $T_{b}$ is the bit duration.

$$
L_{D P I M H C_{\text {avg }}}=\frac{2^{M}+H C_{s}+2 G S H C_{s}}{2 H C_{s}}
$$

\subsection{Differential Amplitude PPMHC (DAPPMHC)}

DAPPMHC is the combination of the differential PPM, PAM and HC. It is used to improve the bandwidth efficiency and TC, however, the power of transmission increased with $k$ number of amplitude. The symbol length $L$ and pulse amplitude $k$ are modulated with HCs. A transmit symbol can be formulated from the set of amplitudes such as $\{1,2, \ldots k\}$, set of slots $\{1,2, \ldots L\}$, and 
HC. $M=\log _{2}\left(L_{(\max ) D A P P M H C} \times k \times H C_{s}\right)$ input bits mapped to one of the distinct waveforms with a pulse indicating at the end of the symbol. Note that DPPMHC can be considered as a special case of DAPPMHC with $k=1$. The average symbol length of DAPPMHC can be express as $L_{D A P P M H C_{\text {avg }}}=\left(2^{M}+k H C_{s}\right) /\left(2 k H C_{s}\right)$.

\subsection{Theoretical Error Performance in Gaussian Channels}

The error probability of PPMHC and DPPMHC can be derived for three constellation points which are above and below the DC bias and zero for empty slots on DC bias as shown in Figure 1. The relation between Bit Error Rate (BER) and slot error can be defined as $B E R=(S E R) M / L$, where, $E_{s}$ is the energy of symbols, which encodes $\log _{2} L$ bits of data.

$$
\sqrt{E_{s}}=L R P_{r} \sqrt{T_{s}}
$$

The probability of slot error for the hard decoding can be derived as:

$$
\begin{array}{r}
P_{e(P P M H C)}=\frac{L-1}{L} Q\left(\frac{\sqrt{E_{s}}}{\sqrt{\frac{\sigma}{2}}}\right)+\frac{1}{L}\left[Q\left(\frac{\sqrt{E_{s}}-\frac{\sqrt{E_{s}}}{2}}{\sqrt{\frac{\sigma}{2}}}\right)\right. \\
\left.+Q\left(\frac{-\sqrt{\frac{E_{s}}{2}}-\left(-\sqrt{E_{s}}\right)}{\sqrt{\frac{\sigma}{2}}}\right)\right] \\
P_{e(P P M H C)}=\frac{L-1}{L} Q\left(\sqrt{\frac{2 E_{s}}{\sigma}}\right)+\frac{2}{L} Q\left(\sqrt{\frac{E_{s}}{2 \sigma}}\right) \\
P_{e(P P M H C)}=\frac{L-1}{L} Q\left(L R P_{r} \sqrt{\frac{2 T_{s}}{\sigma}}\right)+\frac{2}{L} Q\left(L R P_{r} \sqrt{\frac{T_{s}}{2 \sigma}}\right)
\end{array}
$$

The error probabilities for DPPMHC express as

$$
\begin{aligned}
P_{e(D P P M H C)}= & \frac{L_{\text {avg }}-1}{L_{\text {avg }}} Q\left(L_{\text {avg }} R P_{r} \sqrt{\frac{2 T_{s}}{\sigma}}\right) \\
& +\frac{2}{L_{\text {avg }}} Q\left(L_{\text {avg }} R P_{r} \sqrt{\frac{T_{s}}{2 \sigma}}\right) \\
P_{e(D P P M H C)}= & \frac{L_{D P P M H C}}{M} \operatorname{erfc}\left(\sqrt{\frac{M L_{D P P M H C(\text { avg })} E_{b}}{2 \sigma}}\right)
\end{aligned}
$$

In case of PAM, the PNA levels are transmitted. Therefore, the classic trade-off between SER and flickering is considered as per VLC flickering standards ${ }^{16}$. The general form for $k$-PAM for interior and end points symbols of constellation diagram can be derived as follows

$$
\begin{aligned}
P_{s}\left(s_{m}\right)= & \left\{\begin{array}{l}
2 Q\left(\frac{d_{s} / 2}{\sqrt{\sigma / 2}}\right), \text { interior } \\
Q\left(\frac{d_{s} / 2}{\sqrt{\sigma / 2}}\right), \text { end points }
\end{array}\right. \\
P_{s}= & p\left(s_{0}\right) p\left(e \mid s_{0}\right)+p\left(s_{1}\right) p\left(e \mid s_{1}\right)+ \\
& p\left(s_{2}\right) p\left(e \mid s_{2}\right)+p\left(s_{3}\right) p\left(e \mid s_{3}\right) \\
P_{s(4 P A M)}= & \frac{1}{4}\left[\frac{1}{2} \operatorname{erfc}\left(\frac{d_{s}}{2} \sqrt{\frac{E_{s}}{E_{\text {avg }} \sigma}}\right)+\frac{1}{2} \operatorname{erfc}\left(\frac{d_{s}}{2} \sqrt{\frac{E_{s}}{E_{\text {avg }} \sigma}}\right)\right. \\
+ & \left.\operatorname{erfc}\left(\frac{d_{s}}{2} \sqrt{\frac{E_{s}}{E_{\text {avg }} \sigma}}\right)+e r f c\left(\frac{d_{s}}{2} \sqrt{\frac{E_{s}}{E_{\text {avg }} \sigma}}\right)\right] \\
P_{s(4 P A M)}= & \frac{3}{4} \operatorname{erfc}\left(\frac{d_{s}}{2} \sqrt{\frac{E_{s}}{\sigma E_{\text {avg }}}}\right)
\end{aligned}
$$

The end points of every high order PAM on constellation is only two but the interior points could be more for the higher order of PAM. The high order PAM is given in Equation (15)

$$
P_{s(k P A M)}=\frac{k-2}{k} \operatorname{erfc}\left(\frac{d_{s}}{2} \sqrt{\frac{E_{s}}{\sigma E_{\text {avg }}}}\right)
$$

It is analyzed in Equation (15) that, the value of $d_{s}$ can be optimized for different modulation schemes to achieve the minimum acceptable flickering in the room. However, the flickering increases as $d_{s}, k$ increase. Moreover, $d_{s}$ is proportion to SER performance and it also producing flickering in the room.

\subsection{Bandwidth Requirements}

The bandwidth is depending upon the number of slots $L$ in specified symbol duration, and number of HCs. An HC Matrix $(\mathrm{HCM}){ }^{19}$ of order $\left(H C_{s} \times T_{s l}\right)$ can generate the $\mathrm{HC}_{s}$ number of pulses each of $\mathrm{T}_{\text {sl }}$ pulse duration. All the pulses of the HCM are mutually orthogonal. The HCM can exist only if $N=2^{u}$ where $u$ is an integer $(u \geq 0)$. The bandwidth $B$ of each pulse in the HCM is the inverse of shortest time duration peak that is $B_{H C_{s}}=1 / t_{S P \min }$, and every $\mathrm{HC}$ has different bandwidth. Therefore, the average bandwidth is considered. 
In this paper, the bipolar pulses are utilized which are transmitted over and blow the DC bias, therefore, each $\mathrm{HC}$ has two versions of pulses. For example, $H C M_{1 \times 1}$ dimension has two numbers of pulses, and $H_{C M} M_{2 \times 2}$ dimension can generate four numbers of pulses with 1.5 times of average slot bandwidth. Similarly, eight numbers of pulses can be obtained from $H_{C M}$ with average slot bandwidth 2.75 times. The slot bandwidth can be calculated from the duration of shortest peaks of +ve or -ve in HC. Thus, the average bandwidth for a slot can be defined as:

$$
B_{\text {slot(avg) }}=\frac{1}{H C_{s}} \sum_{H C=1}^{H C_{s}} \frac{1}{t_{\text {SPmin }}}
$$

The average bandwidth of PPMHC, DPPMHC, DAPPMHC, and DPIMHC are shown in Equation (18), Equation (19), Equation (20) and Equation (21) respectively.

$$
B=\frac{L}{M} B_{\text {slot (avg) }}
$$

where, $L_{P P M H C}=\frac{2^{M}}{H C_{s}}$

$$
\begin{aligned}
B_{P P M H C} & =\frac{2^{M}}{M H C_{s}} B_{\text {slot (avg) }} \\
B_{D P P M H C} & =\frac{2^{M}+H C_{s}}{2 M H C_{s}} B_{\text {slot (avg) }} \\
B_{D A P P M H C} & =\frac{2^{M}+A H C_{s}}{2 M A H C_{s}} B_{\text {slot (avg) }} \\
B_{D P I M H C} & =\frac{2^{M}+H C_{s}+2 H C_{s} \times G S}{2 M A H C_{s}} B_{\text {slot (avg) }}
\end{aligned}
$$

\subsection{Power Efficiency}

The average optical power emitted by an optical wireless LED is limited due to the eye, skin safety and to keep the portable battery powered consumption to a minimum. The performance criteria for each modulation schemes are power and bandwidth efficiency for indoor optical wireless communication systems. Thus, average optical power and bandwidth are required to achieve the desired SER performance or SNR. Mathematically, power efficiency $\eta_{p}$ is defined as.

$$
\eta_{P}=\frac{E_{\text {pulse }}}{\overline{E_{b}}}
$$

where, $E_{\text {pulse }}$, is the energy per pulse and $\overline{E_{b}}$ is the average energy per bit. The average power requirement for OOK is ${ }^{20}$

$$
P_{\text {avg_ ОоK }}=\sqrt{\sigma R_{b}} Q^{-1}\left(B E R_{\text {ООК }}\right)
$$

Therefore, the average power requirement for PPM (soft decision) is approximately $\underline{21}$

$$
P_{\text {avg_P } P M}=\sqrt{\frac{2 \sigma R_{b}}{L \log _{2} L}} Q^{-1}\left(B E R_{P P M}\right)
$$

The hard decision decoding incurs a $1.5 \mathrm{~dB}$ optical power penalty compared the soft decision decoding. The soft decision can only apply to on the fixed length of symbols, for example, PPM and PPMHC. Therefore, only soft decision decoding is considered. The power efficiency of traditional PPM is given in Equation (25).

$$
\eta_{S_{-} P P M}=\sqrt{\frac{2}{L \log _{2} L}}
$$

Similarly, the average optical power requirements and power efficiency for PPMHC, DPPMHC and DAPPMHC can be express as

$$
\begin{gathered}
\eta_{S_{-} P P M H C}=\sqrt{\frac{2 H C_{s}}{2^{M} \log _{2} \frac{2^{M}}{H C_{s}}}} \\
\eta_{S_{-} D P P M H C}=\sqrt{\frac{8 H C_{s}}{\left(2^{M}+H C_{s}\right) \log _{2}\left(\frac{2^{M}}{H C_{s}}\right)}} \\
\eta_{S_{-} D A P P M H C}=\sqrt{\frac{8 A H C_{s}}{\left(2^{M}+A H C_{s}\right) \log _{2}\left(\frac{2^{M}}{A H C_{s}}\right)}}
\end{gathered}
$$

\subsection{Transmission Capacity}

TC can be defined as the number of possible symbols in the amount of power and bandwidth. It can improve via increasing bit per symbol or by using high bandwidth or transmission power. The TC can be express as $\frac{18}{18}$

$$
C_{T C}=\frac{L_{\max }}{L_{\text {avg }}} \log _{2}\left(2^{M}\right) R_{b}
$$


The TC for PPM is $C_{T C_{-} P P M}=M R_{b}$. Similarly, the TC of PPMHC, DPPMHC, and DAPPMHC can be derived as bellow

$$
C_{T C_{P P M H C}}=M H C_{s} R_{b}
$$

where $L_{\text {max }}=L_{\text {avg }}$ and $H C_{s}>1 L_{\text {avg(DPPMHC })}=\frac{\left(2^{M}+H C_{s}\right)}{2 H C_{s}}$,

$$
L_{\max }=2^{M} / H C_{s} \text { and } H C_{s}>1
$$

$C_{\text {TC_ } D P P M H C}=\frac{M H C_{s} 2^{M+1}}{\left(2^{M}+H C_{s}\right)} R_{b}$

and,

$$
C_{T C_{-} D A P P M H C}=\frac{M A H C_{s} 2^{M+1}}{\left(2^{M}+A H C_{s}\right)} R_{b}
$$

\section{Simulation Result and Discussion}

The setup of the simulation program for indoor VLC system is configured as shown in Figure 2, where the encoder and decoder are used for different modulation schemes.

Figure 3 shows the comparison of PPM, PPMHC, and OOK with HCs (OOKHC) in term of average bandwidth requirement for high order modulation. However, the $\mathrm{HOM}$ of $\mathrm{OOK}$ is achieved by using $\mathrm{HC}_{s}$. Note that, the bandwidth efficiency of PPMHC is better than OOKHC and PPM. Figure 4 is the comparison of bandwidth requirement for DPPM and DPPMHC. The results show that the DPPMHC scheme outperforms the existing scheme DPPM. Note that the average bandwidth requirements for 2 bits per symbol between $\mathrm{DPPrMHC}_{2}$ and $\mathrm{DPPMHC}_{4}$ are same because $B_{\text {slot (avg) }}$ of $\mathrm{DPPMHC}_{4}$ is greater than $\mathrm{DPPMHC}_{2}$. Similarly, in case of DAPPMHC, the average bandwidth of DAPPMHC 2 and DAPPMHC are same at 3 bits/symbols as shown in Figure 5. However, the average bandwidth requirements for $\mathrm{DAPPMHC}_{2}^{2}$ is less than $\mathrm{DAPPMHC}_{4}^{2}$ at 1 and 2 bits per symbol as shown in Figure 5. Similarly, the average bandwidth requirements for $\mathrm{DPPMHC}_{2}^{2}$ is less than $\mathrm{DPPMHC}_{4}^{2}$ at 1 bits per symbol as shown in Figure 4. The bandwidth efficiency of proposed scheme outperforms the existing scheme as shown in Figures 3 to 5.

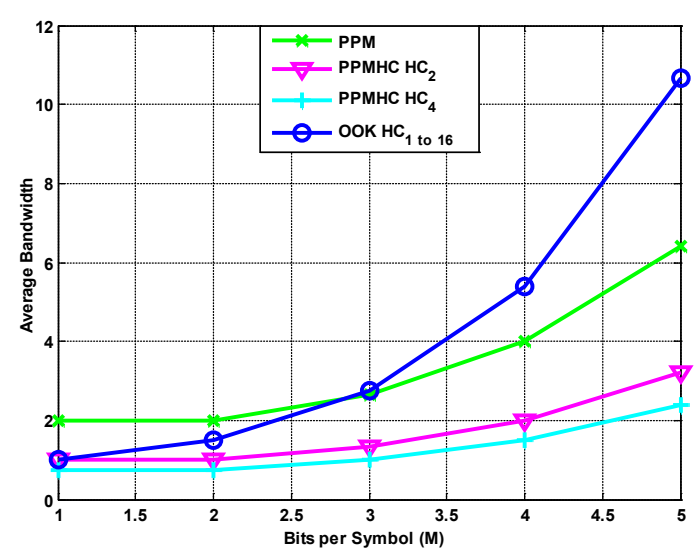

Figure 3. The Average bandwidth required at $M$ bits per symbol for PPM, OOK HC and PPMHC.

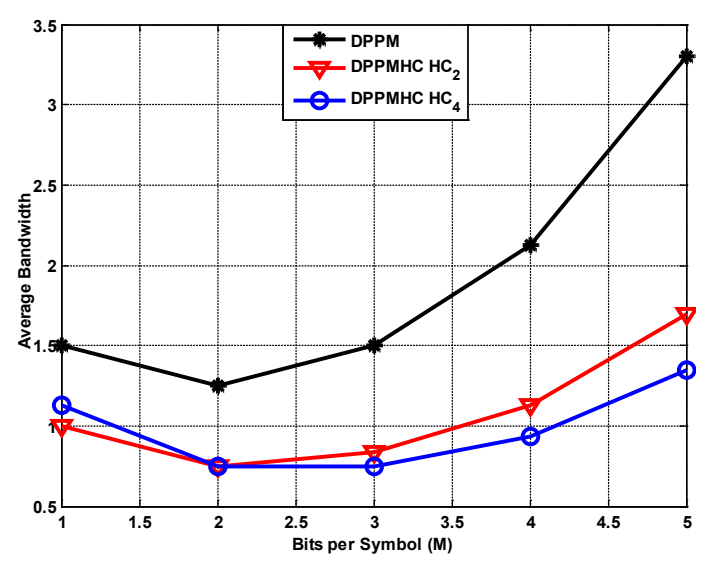

Figure 4. The Average bandwidth required at $M$ bits per symbol for DPPM and DPPMHC.

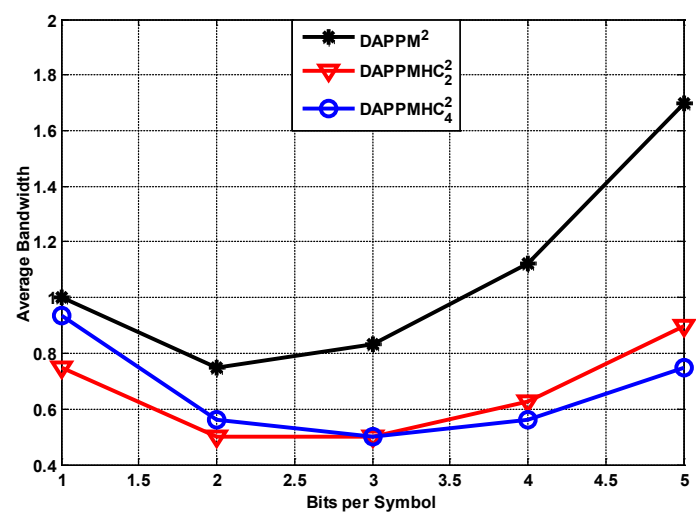

Figure 5. The average bandwidth required at $M$ bits per symbol for DAPPM and DAPPMHC. 
Figures 6-8 show the power and bandwidth required to achieve the $M$ bits per symbol for PPM, PPMHC, DPPM, DPPMHC, DAPPM, and DAPPMHC. The results show that the proposed schemes utilize minor power penalty in time scale, however, it significantly improves bandwidth efficiency and bits rate per transmission.

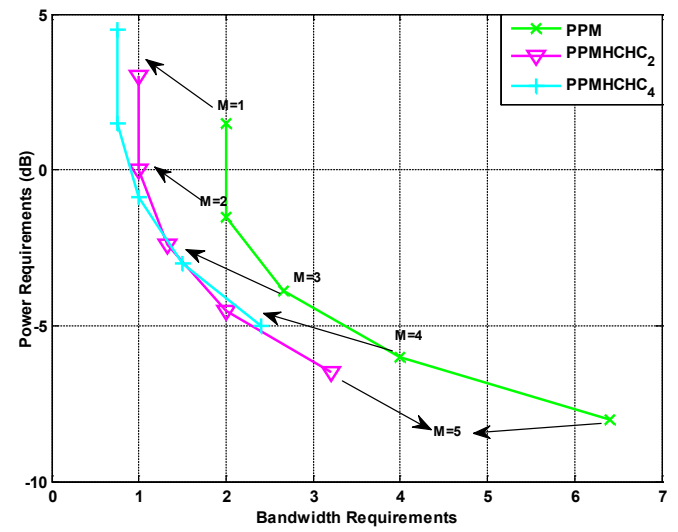

Figure 6. Normalized bandwidth and power requirement of PPM and PPMHC at HOM.

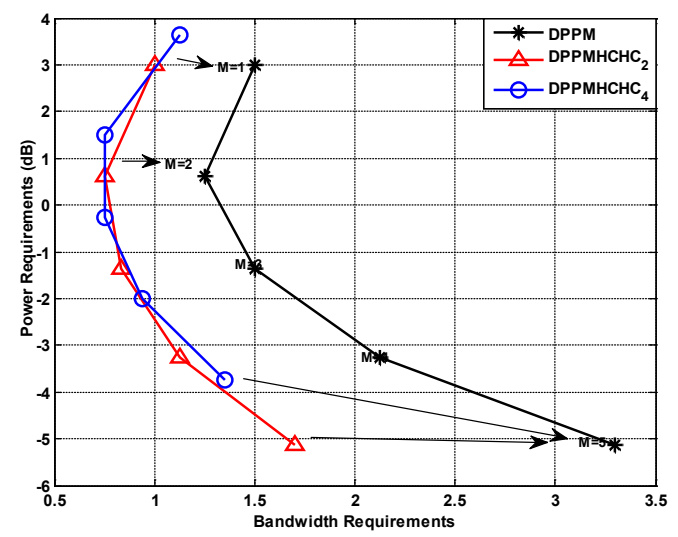

Figure 7. Average bandwidth and power requirement of DPPM and DPPMHC at HOM.

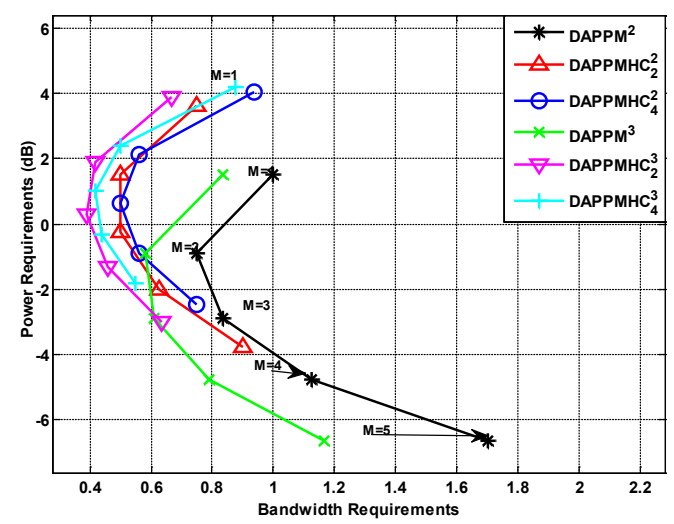

Figure 8. Bandwidth and power requirement of DAPPM and DAPPMHC at HOM.
Figure 9 shows the TC of PPM, DPPM, PPMHC, and DPPMHC at HOM. As depicted in Figure 9, the TC of DPPM, $\mathrm{PPMHC}_{2}$ and $\mathrm{DPPMHC}_{2}, \mathrm{PPMHC}_{4}$ are similar at $M \geq 8$. However, the TC of $\mathrm{DPPMHC}_{4}$ is the double of $\mathrm{DPPMHC}_{2}$, and $\mathrm{PPMHC}_{4}$ and, TC of $\mathrm{DPPMHC}_{2}$ and $\mathrm{PPMHC}_{4}$ is double of DPPM, $\mathrm{PPMHC}_{2}$ at $M \geq 8$. The TC can be further improving by including PAM as shown in Figure 10. Figure 11 is showing the SER performance at different values of $d_{s}$, however, the average transmitted power is same because PNA levels technique is used. The higher values of $d_{s}$ have better SER performances but the flickering problem may occur in the room.

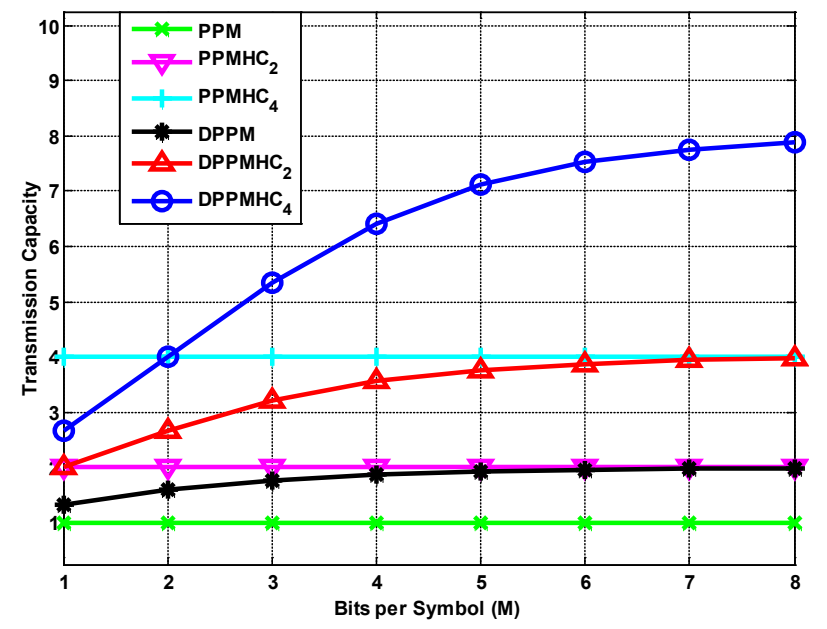

Figure 9. TC of PPM, PPMHC, DPPM, and DPPMHC normalized to PPM vs M.

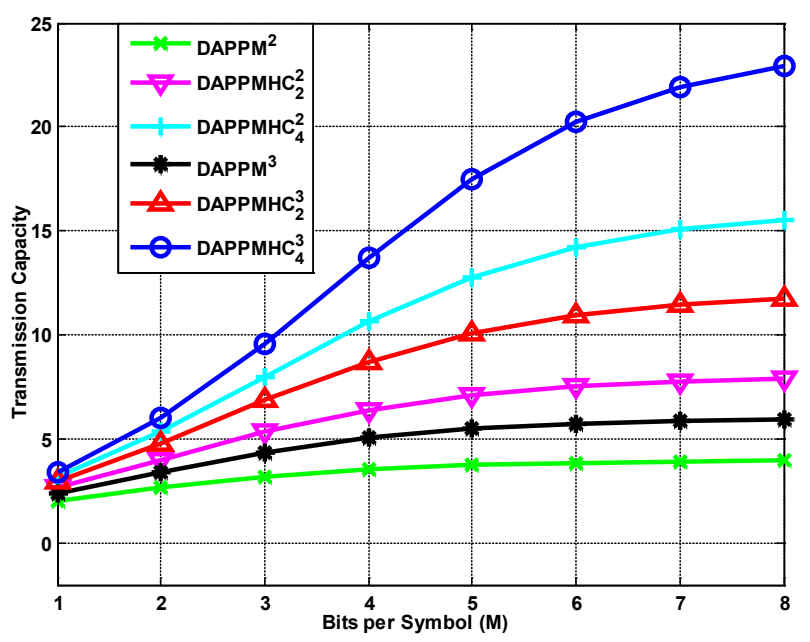

Figure 10. TC of DAPPM and DAPPMHC, at $\mathrm{A}=2,3$. 


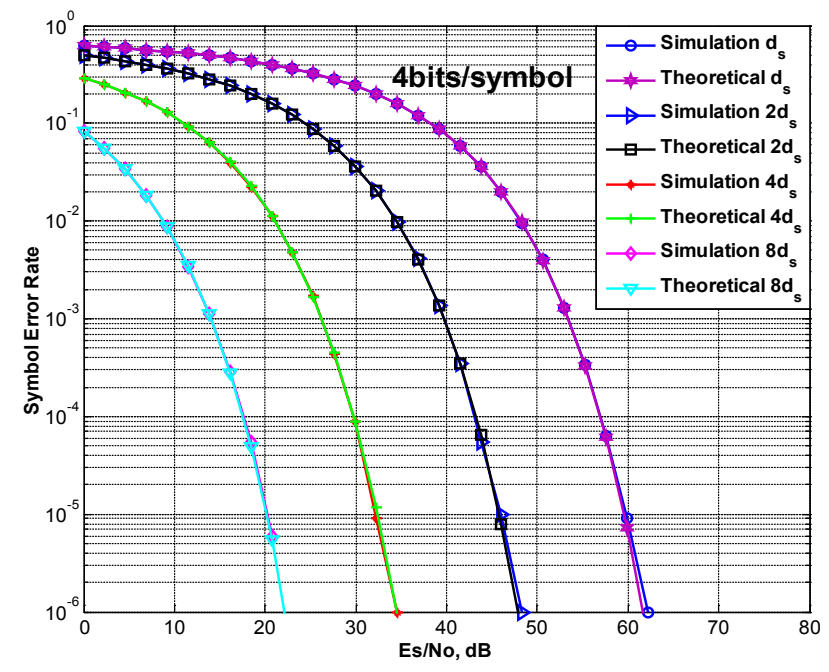

Figure 11. SER comparisons for different values of $d_{s}$.

\section{Conclusion}

In this paper, the improved modulation schemes are presented HCs are combined with PPM, DPPM, and DAPPM to improve the transmission rates, spectral efficiency, and power efficiency. This allows the transmission of more information and better performance at a certain number of orthogonal pulses when compared with conventional modulation techniques. The bandwidth, power, and TC are improved by combining with existing modulation schemes. The HCM generate multiple orthogonal pulses at the cost of higher bandwidth but in this paper, the results show that the proposed scheme outperforms the existing schemes in term of bandwidth, TC, and power efficiency.

\section{Acknowledgement}

The authors would like to thank Southeast University in particularly Prof, Huang Jie in providing research fund for this study.

\section{References}

1. Sethakaset U, Gulliver TA. Differential amplitude pulse-position modulation for indoor wireless optical communications. EURASIP Journal on Wireless Communications and Networking. 2005; 2005:3-11. https://doi.org/10.1155/WCN.2005.3.
2. Komine T, Nakagawa M. Fundamental analysis for visible-light communication system using LED lights. IEEE Transactions on Consumer Electronics. 2004; 50:100-7. https://doi.org/10.1109/TCE.2004.1277847.

3. Ai-Ping H, Yang-Yu F, Yuan-Kui L, Meng J, Bo B, Qing-Gui T. A differential pulse position width modulation for optical wireless communication. 2009 4th IEEE Conference on Industrial Electronics and Applications; 2009. p. 1773-6.

4. Liang Y, Zhang H, Chen L, Liu X, Lin X. A novel low density parity check coded differential amplitude and pulse position modulation free-space optical system for turbulent channel. China Communications. 2017; 14:198-205. https://doi.org/10.1109/CC.2017.7897335.

5. Wang K, Liu B, Zhang L, Zhang Q, Tian Q, Xin X. A novel trellis coded overlapping amplitude and pulse position modulation scheme for gamma-gamma channel free-space optical communication. China Communications. 2016; 13:58-69. https://doi.org/10.1109/CC.2016.7464123.

6. Kong L, Xu W, Zhang H, Zhao C, You X. A PPM-based four-dimensional modulation scheme for visible light communications. 2015 International Conference on Wireless Communications and Signal Processing (WCSP); 2015. p. 1-5. https://doi.org/10.1109/WCSP.2015.7341002.

7. Luna-Rivera J, Perez-Jimenez R, Guerra-Yañez V, SuarezRodriguez C, Delgado-Rajo F. Combined CSK and pulse position modulation scheme for indoor visible light communications. Electronics Letters. 2014; 50:762-4. https:// doi.org/10.1049/el.2014.0953.

8. Rouissat M, Borsai AR, Chikh-Bled M. Isochronous and anisochronous modulation schemes in wireless optical communication systems. International Journal of Information Engineering and Electronic Business. 2012; 4:19. https://doi.org/10.5815/ijieeb.2012.03.03.

9. Mahdiraji GA, Zahedi E. Comparison of selected digital modulation schemes (OOK, PPM and DPIM) for wireless optical communications. 4th Student Conference on Research and Development; 2006. p. 5-10. https://doi. org/10.1109/SCORED.2006.4339297.

10. Yan G, Min W, WeifengD. Performance research of modulation for optical wireless communication system. Journal of Networks. 2011; 6:1099-105. https://doi.org/10.4304/ jnw.6.8.1099-1105.

11. Pergoloni S, Biagi M, Rinauro S, Colonnese S, Cusani R, Scarano G. Merging color shift keying and complementary pulse position modulation for visible light illumination and communication. Journal of Lightwave Technology. 2015; 33:192-200. https://doi.org/10.1109/JLT.2014.2386274.

12. Noshad M, Brandt-Pearce M. Application of expurgated PPM to indoor visible light communications-Part I: Singleuser systems. Journal of Lightwave Technology. 2014; 32:875-82. https://doi.org/10.1109/JLT.2013.2293341. 
13. Yang A, Wu Y, Kavehrad M, Ni G. Grouped modulation scheme for LED array module in a visible light communication system. IEEE Wireless Communications. 2015; 22:24-8. https://doi.org/10.1109/MWC.2015.7096281.

14. Lou S, Gong C, Wu N, Xu Z. Joint dimming and communication design for visible light communication. IEEE Communications Letters. 2017; 21:1043-46. https://doi. org/10.1109/LCOMM.2017.2657740.

15. Ali AY, Zhang Z, Abdelgader AM, Zong B. Data rate optimization in inter-cell interference environment of visible light communication. Photonic Network Communications; 2015. p. 1-9. https://doi.org/10.1007/s11107-015-0585-0.

16. Gomez J, Morcos M. Flicker measurement and light effect. IEEE Power Engineering Review. 2002; 22:11-15. https:// doi.org/10.1109/MPER.2002.1045556.

17. Uddin MS, Cha JS, Kim JY, Jang YM. Mitigation technique for receiver performance variation of multi-color channels in visible light communication. Sensors. 2011; 11:613144. https://doi.org/10.3390/s110606131. PMid:22163946. PMCid:PMC3231439.

18. Ghassemlooy Z, Popoola W, Rajbhandari S. Optical wireless communications: system and channel modelling with Matlab $^{\oplus}$ : CRC Press; 2012.

19. Tang X, Parampalli U. On the noncyclic property of Sylvester Hadamard matrices. IEEE Transactions on Information Theory. 2010; 56:4653-8. https://doi.org/10.1109/ TIT.2010.2053875.

20. Azzam N, Aly MH, AboulSeoud A. Bandwidth and power efficiency of various PPM schemes for indoor wireless optical communications. National Radio Science Conference, NRSC 2009; 2009. p. 1-11.

21. Ramirez-Iniguez R, Idrus SM, Sun Z. Optical wireless communications: IR for wireless connectivity: CRC press; 2008. https://doi.org/10.1201/9781420013443. 